\title{
BMJ Open Acupuncture for migraine: a protocol for a meta-analysis and meta-regression of randomised controlled trials
}

\author{
Zishan Gao, ${ }^{1}$ Carlo Maria Giovanardi, ${ }^{2}$ Hongxiao Li, ${ }^{3}$ Chen Hao, ${ }^{1}$ Qian Li, ${ }^{3}$ \\ Xu Zhang, ${ }^{3}$ Ulrich Mansmann ${ }^{4}$
}

To cite: Gao Z, Giovanardi CM, $\mathrm{Li} \mathrm{H}$, et al. Acupuncture for migraine: a protocol for a meta-analysis and metaregression of randomised controlled trials. BMJ Open 2018;8:e022998. doi:10.1136/ bmjopen-2018-022998

- Prepublication history for this paper is available online. To view these files, please visit the journal online (http://dx.doi org/10.1136/bmjopen-2018022998).

Received 16 March 2018 Revised 15 August 2018 Accepted 5 October 2018

Check for updates

(C) Author(s) (or their employer(s)) 2018. Re-use permitted under CC BY-NC. No commercial re-use. See rights and permissions. Published by BMJ.

${ }^{1}$ Department of Clinical Acupuncture and Moxibustion, Nanjing University of Chinese Medicine, Nanjing, China ${ }^{2}$ Italian Federation of Acupuncture Societies (FISA), Association of Medical Acupuncturists of Bologna (A.M.A.B.), Bologna, Italy ${ }^{3}$ School of Medicine and Life Sciences, Nanjing University of Chinese Medicine, Nanjing, China

${ }^{4}$ Department of Medical Data Processing, Biometrics, and Epidemiology (IBE), LudwigMaximilians-Universität München, Munich, Germany

Correspondence to Dr Ulrich Mansmann; mansmann@ibe.med.unimuenchen.de

\section{ABSTRACT}

Introduction Although the effectiveness of acupuncture for episodic migraine has been confirmed by multiple clinical trials and Cochrane systematic reviews, the mechanisms underlying the specific effect of acupuncture for migraine remain controversial. We aim to evaluate the effectiveness and safety of acupuncture for both episodic migraine and chronic migraine by meta-analysis and explore the possible factors influencing the specific effect of acupuncture for migraine by meta-regression.

Methods and analysis We will search for randomised control trials of acupuncture for migraine in the following eight databases: Cochrane Central Register of Controlled Trials (CENTRAL), MEDLINE, EMBASE, AMED (via OVID) and four Chinese databases (Chinese Biomedical Literature Database, China National Knowledge Infrastructure, Chinese Science and Technology Periodical Database and Wanfang Database) from inception to 31 December 2017. We will also search OpenSIGLE (opensigle.inist. fr) for conference abstracts. No language restriction will be applied. The selection of studies, data extraction and coding and assessment of risk of bias of the included studies will be conducted independently by two reviewers. Standard meta-analysis and, if appropriate, metaregression will be performed using the $\mathrm{R}$ packages Meta and Metafor.

Ethics and dissemination The results of this metaanalysis and meta-regression will be disseminated through publication in a peer-reviewed journal and presented at a relevant conference. The data used in this meta-analysis will not contain individual patient data; therefore, ethical approval is not required.

PROSPERO registration number CRD42018087270.

\section{INTRODUCTION}

\section{Description of the condition}

Migraine is one of the most common headache disorders and is characterised by recurrent unilateral, throbbing, moderate-to-severe attacks of headache lasting from 4 to 72 hours, with or without aura. ${ }^{1}$ Migraine is commonly associated with symptoms of nausea, vomiting, photophobia and phonophobia, and is aggravated by physical exertion. According to the 2010 Global Burden of Disease Survey, migraine ranked as the

\section{Strengths and limitations of this study}

This meta-analysis will not only evaluate the effectiveness of acupuncture in treating both episodic migraine and chronic migraine but also will detect possible characteristics that influence the main effect and the specific effect of acupuncture for migraine.

- Equipped with advanced machine learning techniques and $\mathrm{R}$ packages Meta and Metafor, this meta-analysis could uniquely explore the interaction and combinatory effects of different clinical factors that may affect the main effect and specific effect of acupuncture for migraine.

- The results of this meta-analysis may reveal pivotal factors that impact the clinical effect of acupuncture and thus will support the optimisation of acupuncture treatment for migraine in the pragmatic clinical setting.

- Electronic databases for Korean and Japanese studies will not be searched. Therefore, language bias may exist.

third most predominant disorder, as well as the seventh highest specific cause of disability worldwide. In USA, an estimated 36 million individuals experience migraine attacks, with approximately $16 \%-18 \%$ of women and $6 \%-8 \%$ of men suffering recurrent migraine attacks during their professional lives. ${ }^{2}{ }^{3}$ Migraine usually can be categorised as episodic migraine (migraine attacks happening fewer than 15 days each month) and chronic migraine (migraine attacks happening more than 15 days per month). ${ }^{1}$ Two large population-based studies documented a strong tendency for episodic migraine to evolve into chronic migraine, often associated with overuse of analgesics. ${ }^{4}$ Further, a systematic review focusing on the global prevalence of migraine reported that the prevalence of chronic migraine is $0 \%-5.1 \%$, with estimates typically among $1.4 \%-2.2 \% .^{5}$ Recently, many studies also demonstrated a correlation between migraine and cardiovascular and 
cerebrovascular diseases. ${ }^{6} 7$ A systematic review investigating migraine and cardiovascular disease found that the risk of ischaemic stroke was doubled in people who had migraine with aura. ${ }^{8}$ It is well accepted that migraine with aura is associated with ischaemic stroke in younger women (age $\leq 45$ years) ${ }^{9}$

\section{Description of the intervention}

To be considered effective, treatments for migraine should reduce the occurrence of migraine attacks and relieve symptoms in order to improve patients' quality of life and decrease related healthcare utilisation. Pharmacological agents for migraine are widely used to reduce migraine attack frequency and pain intensity, including propranolol, metoprolol, flunarizine, valproic acid and topiramate. ${ }^{10}$ Unlike acute migraine that can often be managed with drugs, chronic migraine is difficult to control due to the frequency of attacks and overuse of drugs among migraine patients. ${ }^{5}$ Furthermore, pharmacological treatment for migraine with oral nonsteroidal anti-inflammatory drugs and tripans has a modest effect and often leads to several side effects, such as gastrointestinal and cardiovascular disorders. ${ }^{11}$ Thus, there is a strong need for the development and implementation of additional long-term effective, low-risk treatments for migraine.

Acupuncture is a procedure whereby fine needles are inserted into and manipulated within the skin of acupoints at certain depths to reduce symptoms and achieve curing of diseases. ${ }^{12}$ Acupuncture originated in China within the Chinese civilisation and has become one of the most widely used forms of traditional Chinese medicine (TCM). The treatment effect of acupuncture is regarded as balancing the disorder within the human body in order to treat diseases. Such balancing is achieved by activating accurate meridians and acupoints of the body using diverse needling techniques, according to disease and personal status. Currently, even without a clear understanding of underlying mechanisms, acupuncture has been extensively used for migraine prophylaxis and chronic pain treatment worldwide. According to a survey in USA, $9.9 \%$ of patients who received acupuncture treatment did so in search of relief from migraine and other headaches. ${ }^{13}$ A 2016 Cochrane Review from Germany concluded that acupuncture is safe and effective for episodic migraine prophylaxis compared with prophylactic drug treatment. ${ }^{14}$

\section{How the intervention might work}

While enhanced calcitonin gene-related peptide (CGRP) and spontaneous cortical spreading depression are regarded as causative in triggering migraine,${ }^{15}$ the pathophysiology of migraine is attributed to multiple factors, many of which remain unclear. Recently, an increasing number of studies have confirmed that acupuncture activates the release of opioid peptides in the central nervous system (CNS). Release of these peptides corresponds to long-lasting activation of ascending sensory tracks, thereby relieving an array of pain conditions. ${ }^{16-19}$ Furthermore, Zhao et al confirmed in a migraine rat model that electro-acupuncture treatment can depress CGRP expression in the trigeminal ganglion, which plays a key role in inducing migraine attack. ${ }^{20} \mathrm{Li}$ et al also found that acupuncture restores the impaired descending pain modulatory system in migraine patients by decreasing the resting state functional connectivity between the periaqueductal grey and rostral anterior cingulate cortex/ medial prefrontal cortex, which directly correlated with the intensity of pain during the migraine attack. ${ }^{21}$

In contrast to the conflicting evidence concerning the mechanism by which acupuncture relieves migraine, evidence of the effectiveness of acupuncture for migraine was provided by a series of Cochrane systematic reviews and a large individual patient meta-analysis. ${ }^{1422}$ However, the concept of a 'placebo effect' and 'specific effect' regarding the clinical effect of acupuncture for migraine is still controversial. ${ }^{22}$ An updated Cochrane review of acupuncture for episodic migraine concluded that acupuncture is an effective treatment for the prevention of migraine, but on the other hand, the difference in the effect size between acupuncture and sham acupuncture was relatively small. ${ }^{14}$ Linde $e t$ al argued that understanding the specific effect of acupuncture is essential for the acceptance of acupuncture as a legitimate treatment in Western countries. Therefore, evidence confirming the specific effect of acupuncture is still in high demand for biomedicine. ${ }^{23}$ Nevertheless, key components of the effect of acupuncture remain to be fully uncovered. Accordingly, designing appropriate sham acupuncture as a placebo control is still a difficult task for clinical acupuncture trials. Thus, this debated issue may be an obstacle to the acceptance of acupuncture as a valid therapy in pain management by both Western scientific researchers and policymakers.

\section{Why it is important to do this review}

To investigate the effect and specificity of acupuncture, a newly updated Individual Patient Data Meta-Analysis of acupuncture for chronic pain ${ }^{24}$ concluded that true acupuncture has a long-term effect compared with sham acupuncture. Nevertheless, it also pinpointed that characteristics of acupuncture treatment sessions contribute to the effect of acupuncture, in addition to the specific effects of needling. However, this meta-analysis mainly focused on chronic pain and chronic headache and was not specific to migraine. There is still insufficient evidence to elucidate the specific mechanism of the effect of acupuncture for migraine. Furthermore, a recent German Cochrane Review ${ }^{14}$ demonstrated the effectiveness of acupuncture in the prevention of episodic migraine. Whereas there has been no systematic review of the effect of acupuncture in the prevention of chronic migraine. Therefore, three pivotal questions arise from the former meta-analysis and clinical studies of acupuncture for migraine: (i) is acupuncture an effective therapy for both episodic migraine and chronic migraine in the 
clinical setting? (ii) are there any important factors that influence the effect of acupuncture? and (iii) are there any features that alter the specific effect of acupuncture compared with sham acupuncture or other controls in clinical acupuncture treatment for migraine? To address these critical questions, we will conduct a meta-analysis of randomised control trials (RCTs) of acupuncture for migraine.

\section{Objective}

This meta-analysis in adults with episodic or chronic migraine aims to assess the following aspects:

1. The effectiveness and safety of acupuncture compared with sham acupuncture and drug treatment or waiting list controls in the prevention of migraine.

2. The features that may contribute to (i) different effect sizes between acupuncture and controls, (ii) the specific effect of acupuncture, (iii) the non-specific effects of sham acupuncture or other controls and to identify (iv) which factors among all those studied most strongly influence the effect of acupuncture.

\section{METHODS}

\section{Criteria for considering studies for this review}

Types of studies

We will only include RCTs investigating the effect of acupuncture on episodic and chronic migraine in adults. ${ }^{4}$ We will only include RCTs (parallel groups as well as crossover) using well-described randomization methods.

We will exclude quasi-randomised clinical trials.

\section{Types of participants}

We will include patients of either sex, aged 18 and older who have been diagnosed either with episodic or chronic migraine. Migraine diagnoses must be based on the International Classification of Headache Disorders (ICHD-III beta 2013 and its previous editions ICHD-II 2004) proposed by the International Headache Society (IHS 1988) and the Ad Hoc Committee on the Classification of Headache Ad Hoc 1962. ${ }^{125-27}$ If no specified criteria were documented in the studies, the migraine diagnosis must be based on discriminable and important characteristics of migraine attacks (eg, recurrent headache, unilateral pain, pulsating quality, moderate or severe intensity, in association with nausea and/or photophobia and phonophobia), as confirmed by patients' doctors or general practitioners. In general, episodic migraine occurs in two to eight episodes but with migraine attacks less than 15 days per month, whereas chronic migraine patients usually suffer migraine attacks at least 15 days per month (180 days per year) for at least 3 months. ${ }^{1}$

The duration of migraine history must be longer than 1 year in the majority of patients. This criterion will be considered met if

1. An explicit description of the duration of migraine history longer than 1 year is noted in the inclusion criteria.
2. The mean duration minus one SD is longer than 1 year as shown in the table of baseline characteristics.

3. Other information confirms that the criterion was met (eg, proportions with duration ranges are presented in the studies).

We will include studies in which patients were defined as having 'combination' or 'mixed' migraine only if we can extract the data on participants suffering from migraine.

We will exclude trials including patients with secondary headache. Studies including chronic migraine patients with medication-overuse history (according to the ICHD, third beta edition (ICHD-IIIß) criteria $^{26}$ ) will also be excluded.

\section{Types of intervention}

\section{Experimental intervention}

We define acupuncture (both manual and electrical stimulation) as the experimental intervention. Acupuncture is defined as insertion of specific needles into the skin of the body at selected acupoints (defined as 'meridian acupoints', which belong to 14 meridians in the body according to traditional acupuncture theory), pain points (defined as 'a-shi points' in the location of pain condition according to traditional acupuncture theory) or extraordinary points (defined as 'extra points', which do not belong to the 14 meridians but have a therapeutic effect in the body according to traditional acupuncture theory) up to definite therapeutic depths. In accordance with a previous Cochrane systematic review of acupuncture for migraine, ${ }^{14}$ the dosage of acupuncture treatment must be at least six treatment sessions, with a duration of at least $20 \mathrm{~min}$ per session and at least one session per week in the majority of patients. Also, for the purpose of ensuring the clinical effectiveness of acupuncture treatment, the acupuncturists who administered treatment in the included studies should be confirmed to have a relevant acupuncture qualification or professional affiliation, or a certain number of years in acupuncture practice. In addition, trials that define acupuncture in combination with other pharmacological treatment or physical treatment as the experimental intervention but mainly investigate the effectiveness of acupuncture will also be included.

As this meta-analysis will mainly focus on the effectiveness of acupuncture on the basis of traditional acupuncture theory and the Standards for Reporting Interventions in Clinical Trials of Acupuncture (STRICTA), ${ }^{28}$ we will exclude trials in which:

1. Acupuncture was performed at one specific body area but not at acupoints of the body, such as scalp acupuncture, ear acupuncture and wrist-ankle acupuncture.

2. Acupoints were stimulated by other techniques without traditional acupuncture needling, including acupressure, laser stimulation, injection acupuncture, dry needling and trigger point therapy.

\section{Control interventions}

We will include three types of control interventions: 
1. No treatment or waiting list-control during the trial period.

2. Sham acupuncture (intervention resembling verum acupuncture treatment but using superficial needle insertion, needle insertion at non-acupuncture points or at points not indicated for the condition under study and 'placebo' needles that seem to be inserted into skin but actually are not ${ }^{29-32}$ etc). Trials that have intervention groups comparing either acupuncture alone with sham intervention alone or acupuncture plus one or more therapies with sham intervention plus the same therapies also will be included.

3. Pharmacological treatment that is given as a control during a comparable time as application of acupuncture treatment.

Because our objective is to evaluate the effectiveness of acupuncture treatment compared with sham acupuncture treatment, no treatment or western medicine treatment, we will exclude trials with herbal medicine, moxibustion, bloodletting and other different forms of acupuncture as control interventions.

\section{Types of outcome measures}

To be considered for inclusion, trials must have evaluated at least one of the following primary efficacy outcome measures $^{33}$ for at least 4 weeks from the beginning of acupuncture treatment:

1. Number of migraine attacks per evaluation interval.

2. Number of migraine days per evaluation interval.

Before the review process, a review board consisting of an epidemiologist, acupuncturist, migraine patient, social policymakers and statistician will be established to determine all the key outcomes with respect of migraine patients' opinions and values.

We will exclude studies that

1. Included primary outcome measurements only using 'effectiveness rate'.

2. Exclusively used objective or surrogate outcome measures.

3. Evaluated treatment or measurement of acute migraine attack.

4. Had outcome evaluation periods shorter than 4 weeks (after randomization to end of treatment).

According to the guidelines for controlled trials of drugs in migraine published by theIHS, ${ }^{33}$ the main outcomes will be the following.

\section{Primary outcome}

Migraine frequency:

We will consider the following outcomes measuring headache frequency:

1. Numbers of migraine attacks per evaluation interval.

2. Number of migraine days per evaluation interval.

\section{Secondary outcomes}

1. Migraine intensity: outcomes recording pain intensity using numerical/verbal scale, such as average headache severity per evaluation interval.
2. Responder rate (patients with $\geq 50 \%$ reduction in headache frequency) per evaluation interval.

3. Medication intake for migraine per evaluation interval.

4. Adverse events, including the number of patients who dropped out due to an adverse event and the number of patients who reported adverse events.

In contrast to a previous meta-analysis of acupuncture for migraine prophylaxis, we define responder rate as a secondary outcome. According to the IHS guidelines, ${ }^{33}$ the responder rate is comparatively insensitive to the treatment effect and particularly vulnerable to selection bias. The responder rate can be evaluated as an important secondary outcome in placebo-controlled RCTs of migraine.

Outcome measurement may be performed at specific time points; the choice will depend on the time when the outcomes are reported in the reviewed studies. Specific decisions will be made by the review board.

The following outcome measures will be presented in the' Summary of findings' table:

1. Migraine frequency.

2. Migraine intensity.

3. Responder rate.

4. Medication use for migraine attacks.

5. Adverse events.

\section{Patient and public involvement}

Patient and public involvement will be considered during the entire meta-analysis. We collected patients' suggestions and comments from both China and Italy for the selection of outcomes and design of this meta-analysis. In addition, we will collaborate with the Italian Federation of Acupuncture Societies to collect novel evidence for the application of acupuncture for migraine and establish a long-term medical collaboration through the European Union's Seventh Framework Programme (FP7/2007-2013) under REA grant agreement number PIRSES-GA-2013-612 589: CHETCH (China and Europe Taking Care of Healthcare solutions). Thus, our findings will be regularly disseminated to both Chinese and European residents by the local medical institutions.

\section{Search methods for identification of studies}

We will conduct our meta-analysis in accordance with the Cochrane Handbook for Systematic Reviews of Interventions ${ }^{34}$ and will report this meta-analysis based on the PRISMA (Preferred Reporting Items for Systematic Reviews and Meta-analyses) guidelines.

\section{Electronic searches}

We will search the Cochrane Central Register of Controlled Trials, MEDLINE, EMBASE and AMED (via OVID) databases as well as four Chinese databases (Chinese Biomedical Literature Database, China National Knowledge Infrastructure, Chinese Science and Technology Periodical Database, and Wanfang Database) from inception to 31 December 2017. No language restriction will be applied. The reference lists of retrieved 


\begin{tabular}{|c|c|}
\hline Number & Search terms \\
\hline 1 & Headache disorders [MeSH] \\
\hline 2 & Headache[MeSH] \\
\hline 3 & $\begin{array}{l}\text { (headache or migraine or cephalgia or cephalalgia } \\
\text { or chronic migraine):ti,ab (Word variations have } \\
\text { been searched) }\end{array}$ \\
\hline 4 & 1 or 2 or 3 \\
\hline 5 & Acupuncture therapy (MeSH) \\
\hline 6 & $\begin{array}{l}\text { (acupuncture or electroacupuncture or electro- } \\
\text { acupuncture): ti,ab }\end{array}$ \\
\hline 7 & 5 or 6 \\
\hline 8 & randomised:ti, ab. \\
\hline 9 & randomized:ti,ab. \\
\hline 10 & randomly:ti,ab. \\
\hline 11 & placebo:ti,ab \\
\hline 12 & Clinical trials $[\mathrm{MeSH}]$ \\
\hline 13 & trial: ti,ab. \\
\hline 14 & randomized controlled trial [MeSH]:ti,ab. \\
\hline 15 & randomised controlled trial [MeSH]:ti,ab. \\
\hline 16 & controlled clinical trial:ti,ab \\
\hline 17 & 8 or 9 or 10 or 11 or 12 or 13 or 14 or 15 or 16 \\
\hline 18 & Humans \\
\hline 19 & 17 and 18 \\
\hline 20 & 4 and 7 and 19 \\
\hline
\end{tabular}

trials and previous systematic reviews will be searched for citation of potentially eligible trials. We will contact the corresponding author of articles, if any questions about trials arise.

The search strategy for MEDLINE is shown in table 1.

\section{Searching other resources}

We will search the US National Institutes of Health Ongoing Trials Register (http://www.clinicaltrials.gov), the WHO International Clinical Trials Registry Platform (http://www.who.int/trialsearch) and the metaRegister of Controlled Trials (http://www.controlledtrials.com) for any relevant ongoing or unpublished trials. OpenSIGLE (opensigle.inist.fr) will be searched for conference abstracts. We will also search Google Scholar ( scholar.google.com/advanced scholar search?hl=en\&lr=) using the search string 'acupuncture AND (headache OR migraine OR chronic migraine)' for potentially relevant trials from inception to 31 December 2017.

\section{Data collection and analysis}

Selection of studies

Two independent reviewers will examine titles and abstracts of the identified studies and will exclude irrelevant trials. When the first selection is made, full articles will be obtained and checked again in more detail. Following this assessment, a second selection will be performed. The criteria for both selections will be extracted and documented. Possible conflicts will be resolved by discussion, which will also include a third reviewer. The selection process will be presented in a PRISMA flow diagram (figure 1).

\section{Data extraction and coding}

Two independent reviewers will extract data from the selected studies using pilot-tested data forms. They will include the following study information: author, year of publication, study populations (European ancestry or not), study design, numbers of patients randomised and treated, number of patients analysed, baseline analysis, random sequence generation, allocation concealment method, blinding method, imputation method, withdrawals of data, interventions, controls, medication records, and primary and secondary outcomes at all reported time points. For investigating the characteristics of acupuncture effect, we will extract data on age, sex, populations, headache classifications, number and duration of treatment sessions, features of acupuncture treatment (such as type of acupuncture, needle depths, selection of points, achievement of de-chi, manipulation between acupuncture treatment or not), features of control interventions (sham methods, drug use, or standard treatment details), patients' expectations and experience of acupuncturists in accordance with STRICTA. ${ }^{28}$

We also will document for each outcome of the percentage of missing values reported in the study.

For the purpose of analysing the influence of characteristics of acupuncture on its effect size, a coding sheet will be developed to transform all the described data into categorical data. Pilot testing on this coding sheet will be performed on a separate subset of studies. A coding book will be subsequently established to guide the coding process when the code sheet is completed. Two independent reviewers and statisticians will check the coding sheet when coding process has been finished. ${ }^{35}$

\section{Assessing risk of bias in included studies}

Two reviewers will independently assess the risk of bias for each included RCTs using the Cochrane Collaboration's risk of bias tool. ${ }^{34}$ The critical assessment for the risk of bias will be evaluated in seven domains: random sequence generation, allocation concealment, blinding of participants and personnel, blinding of outcome assessment, incomplete outcome data, selective reporting and other sources of bias. This assessment will be achieved by assigning a judgement of 'low risk' of bias, 'high risk' of bias or 'unclear risk' of bias. Because we will only include RCTs using well-described randomisation, only those RCTs considered to have a low risk of bias for random sequence generation will be included. Any disagreement will be resolved by discussion or consensus with a third reviewer. The graphical presentation of assessment of risk of bias will be generated by RevMan V.5.3.5. 


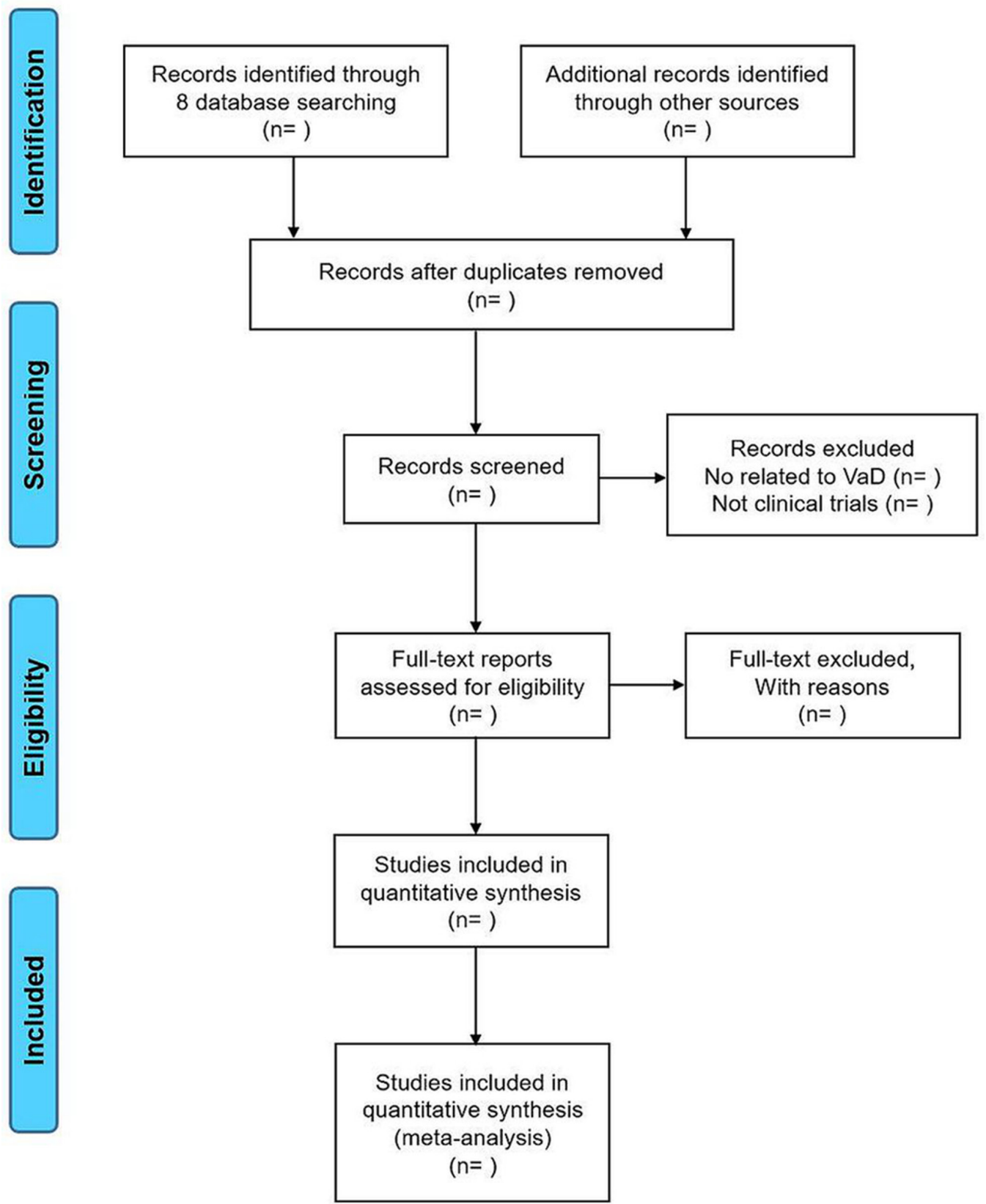

Figure 1 Preferred Reporting Items for Systematic Review and Meta-Analysis (PRISMA) 2009 flow diagram.

\section{Measures of treatment effect}

To address the clinical effect difference between the intervention and control groups, headache frequency at the completion of treatment and at the end of follow-up will be used as a primary outcome. Pain intensity, responder rate and mediation intake at the completion of treatment and at the end of follow-up also will be extracted as secondary outcomes. For these continuous outcomes, the mean difference (MD) and SDs will be extracted and calculated as an effect estimate. Negative values will indicate better outcomes in the acupuncture group.

If the MD or SDs were not reported and not available after contacting the authors, we will use the data that are available, such as the median or $p$ values and confidence intervals, and try to re-calculate MD and SD values from the information recorded in the study.
The safety or adverse outcome will be the number of participants who dropped out due to adverse effects and the number of participants who reported at least one adverse event or effect. For these dichotomous outcomes, the OR will be calculated as the effect estimate. An OR greater than 1 will indicate more events (eg, dropouts) in the acupuncture group.

For the time window analysis, we will extract outcomes with all the time points using pilot-tested data forms. Subsequently, we will document the quantitative outcomes at the end of treatment together with the length of the treatment period. In a meta-regression, we will adjust treatment effects for the time. The $\mathrm{R}$ package Metafor will allow us to give each outcome a meta-analytic result at different treatment periods. Standard meta-analyses, 
especially for subgroups, will be performed using the R-package Meta. ${ }^{3637}$

\section{Unit of analysis issues}

The unit of analysis will be based on aggregated outcome data due to the lack of individual patient data.

\section{Dealing with missing data}

If there are insufficient details or missing data in relation to the characteristics of the studies included in the meta-analysis, we will attempt to contact the study authors for further information first. For missing participant data due to dropout or loss to follow-up, we will apply the following strategies to address missing data assumed to be not missing at random:

1. If intention to-treat (ITT) analyses were performed in the included studies, we will use the ITT data instead of missing data as the first option.

2. For continuous missing outcome data, we will try to re-calculate MD and SD values as the first option when the medians, $p$ values or confidence intervals are reported in the included studies.

3. If there are no ITT data or possible data for re-calculation, we will perform a sensitivity analysis to elucidate the influence of missing data on the effect estimates as a second option. This can be performed by a meta-regression adjusting for the amount of missing data.

\section{Assessment of heterogeneity}

We will evaluate the heterogeneity of the included studies with $\mathrm{I}^{2}$ statistic and the tau ${ }^{2}$ test. A cut-off point of at least $50 \%$ for the $\mathrm{I}^{2}$ statistic will indicate substantial heterogeneity.

Our second aim is to investigate which clinical setting and which acupuncture features do influence the effect size and the heterogeneity of the intervention. First, we will define characteristics that may modify the intervention effect according to experienced acupuncturists. These acupuncturists are required to have a qualified acupuncture license and at least 10 years of clinical acupuncture experience in accordance with STRICTA. ${ }^{28}$ Second, we will examine the correlation between these covariates to exclude possible masking and to establish a core set of covariates. A random-effects meta-regression analysis will be conducted using metafor ${ }^{36}$ to elucidate the impact of core set covariates on treatment effects.

Before the meta-analysis can be conducted, the relevant results from each study must be quantified in such a way that the resulting values can be further aggregated and compared. Depending on different aspects (goals of the meta-analysis, the design and types of studies included and the information provided therein), we will calculate the effect size of interest using the escalc function.

Subsequently, random/mix-effects of the meta-regression model will be fitted by the rma() function. ${ }^{36}$ The restricted maximum-likelihood estimator, which is an approximately unbiased and efficient estimator, will be used to address the amount of residual heterogeneity $\operatorname{tau}^{\wedge 2}$. The pre-defined covariates (eg, characteristics of the acupuncturists) will be first fitted independently to examine the possible independent factors contributing to variation in the intervention effect. Subsequently, interaction between covariates (eg, characteristics of the acupuncturists and session of acupuncture treatment) can be added and detected in the model using the mods argument. For the limit of included studies, we will put covariates $\leq 3$ into the same model for each model. For the result interpretation, the estimate represents the average effect estimates of covariates and a $p$ value $\leq 0.05$ represents a significant difference that indicates the corresponding covariate plays an important role for the intervention effect and heterogeneity. In addition, the amount of heterogeneity in the effect estimates will be estimated by $\operatorname{tau}^{\wedge^{2}}$. The $\mathrm{I}^{2}$ statistic estimates (in percent) describe that how much of the total variability in the effect size estimate (which is composed of heterogeneity and sampling variability) can be attributed to heterogeneity among the true effects. The results of the meta-regression outlined above will be presented in a series of summary tables in the meta-analysis.

\section{Assessment of reporting biases}

Reporting bias will be explored by constructing funnel plots and performing Egger's test, if there are at least 10 trials included in meta-analysis.

\section{Data synthesis}

The synthesis will be done by generating a forest plot for meta-regression. This plot does not contain a summary measure given by a prism below the single studies, but by a prism shown for each single study that shows the aggregated effect for the specific type of study (depending on the covariates of the meta-regression). If the heterogeneity test indicates there is no substantial heterogeneity between studies, the Mantel-Haenszel method implemented by the rma.mh() function will be fitted for calculating pooled estimates, 95\% CIs and combined $\mathrm{p}$ values. If substantial heterogeneity is indicated by $\mathrm{I}^{2} \geq 50 \%$, the random-effects model will be performed by the DerSimonian and Laird method (DerSimonian 1986) and the rma function. The significance of the $p$ value represents the strength of evidence against the null hypothesis of no intervention effect.

\section{Subgroup analysis}

Subgroup analysis will be performed according to the primary and secondary objectives. To detect possible heterogeneity of the results, subgroup analysis will be conducted for both the primary outcome and secondary outcomes at the end of the treatment session and the end of the follow-up period. We will investigate the effects in four subgroup analyses:

1. Episodic migraine vs chronic migraine.

2. Acupuncture vs different type of sham acupuncture and controls.

3. Western studies vs Chinese studies. 
4. Early time-point of outcomes vs later time-point of outcomes.

In addition, if we detect any important and significant covariates contributing to the variation of the intervention effect by meta-regression, subgroup analyses will also be conducted according to these covariates.

\section{Sensitivity analysis}

To confirm the robustness of our findings, a sensitivity analysis will be conducted based on the different levels of bias of the included studies. To evaluate the internal validity of studies or treatment adequacy, we will subsequently remove studies of 'high risk' of bias, studies of 'unclear risk' of bias, and studies of 'low risk' of bias using the metafor package and leavelout function.

\section{Summary of evidence}

We will summarise the quality of evidence using the GRADE (Grading of Recommendations Assessment, Development and Evaluation) approach ${ }^{38}$ and present 'Summary of findings' tables. The 'Summary of findings' tables will be generated by the GRADE working group software (GRADEpro or GRADEpro GDT (www. gradepro.org)). The content of the 'Summary of findings' tables (main outcomes that are important to patients and decision makers) will be determined by the review group described above. Where possible, both relative and absolute measures of effect will be provided. To assess the quality of evidence, the GRADE approach evaluates the quality of evidence as 'high', 'moderate', 'low', or 'very low' by the outcome. Evidence can be downgraded in category by concerns about risk of bias, imprecision, inconsistency, indirectness or publication bias, and also can be upgraded by a large effect size, plausible confounding that could change the effect size and dose-response relation. Reviewers will downgrade or upgrade the evidence according to the GRADE guideline in the Cochrane handbook, Chapter $11^{34}$ and also take into account the differences in anticipated effects in the group of primary interest. The total quality of the evidence will be adjudicated on the basis of both reviewers and all the members of the review board.

\section{Ethics and dissemination}

The results of this meta-analysis and meta-regression will be disseminated through publication in a peer-reviewed journal and be presented at a relevant conference. The data that will be used will not contain individual patient data; therefore, there are no concerns about patients' privacy.

\section{DISCUSSION}

This meta-analysis will not only evaluate the evidence from published RCTs for the effectiveness of acupuncture in treating both episodic migraine and chronic migraine, but also will detect possible characteristics that influence the main effect and the specific effect of acupuncture for migraine. This will be achieved by using meta-regression. We hope that using meta-regression techniques in this meta-analysis will not only provide a deeper understanding of the effect of acupuncture in patients with migraine, but also generate evidence for factors that modify the effect, which will support the optimisation of acupuncture treatment for migraine in the pragmatic clinical setting. If this protocol must be amended, we will present the date of each amendment with a description of the change and the corresponding rationale.

Acknowledgements The authors are grateful to Dr Alessandra Poini and Dr Umberto Mazzanti for their helpful assistance.

Contributors UM is the guarantor. ZG, UM, CMG and XZ contributed to the conception of the study. The manuscript presenting the protocol was drafted by ZG and revised by UM. The search strategy was developed by all authors and will be run by $Z G$ and $H L$, who will also independently screen the potential studies, extract data from included studies and assess the risk of bias. ZG and UM will conduct and finish the data synthesis. QL and $\mathrm{CH}$ will arbitrate in cases of disagreement and ensure no errors occur during the study. All authors have approved the publication of the protocol.

Funding This meta-analysis will be supported by funding from the National Natural Science Foundation of China (number 81202741), the Research Fund for the Doctoral Program of Higher Education of China (number 20123237120009), the State Scholarship Fund (number 201608320044) and the People Programme (Marie Curie Actions) of the European Union's Seventh Framework Programme FP7/20072013/ under REA grant agreement number PIRSES-GA-2013-612 589: CHETCH (China and Europe Taking Care of Healthcare solutions).

Competing interests None declared.

Patient consent Not required.

Ethics approval Ethical approval is not required.

Provenance and peer review Not commissioned; externally peer reviewed.

Open access This is an open access article distributed in accordance with the Creative Commons Attribution Non Commercial (CC BY-NC 4.0) license, which permits others to distribute, remix, adapt, build upon this work non-commercially, and license their derivative works on different terms, provided the original work is properly cited, appropriate credit is given, any changes made indicated, and the use is non-commercial. See: http://creativecommons.org/licenses/by-nc/4.0/.

\section{REFERENCES}

1. Headache Classification Committee of the International Headache Society (IHS). The international classification of headache disorders, 3rd edition (beta version). Cephalalgia 2013;33:629-808.

2. Fumal A, Schoenen J. Current migraine management - patient acceptability and future approaches. Neuropsychiatr Dis Treat 2008;4:1043-57.

3. Stovner LJ, Andrée C. Impact of headache in europe: a review for the eurolight project. $J$ Headache Pain 2008;9:139-46.

4. Kristoffersen ES, Lundqvist C. Medication-overuse headache: epidemiology, diagnosis and treatment. Ther Adv Drug Saf 2014;5:87-99.

5. Natoli JL, Manack A, Dean B, et al. Global prevalence of chronic migraine: a systematic review. Cephalalgia 2010;30:599-609.

6. Adelborg K, Szépligeti SK, Holland-Bill L, et al. Migraine and risk of cardiovascular diseases: Danish population based matched cohort study. BMJ 2018;360:k96.

7. Kurth $\mathrm{T}$, Winter AC, Eliassen $\mathrm{AH}$, et al. Migraine and risk of cardiovascular disease in women: prospective cohort study. BMJ 2016;353:i2610.

8. Schürks M, Rist PM, Bigal ME, et al. Migraine and cardiovascular disease: systematic review and meta-analysis. BMJ 2009;339:b3914.

9. Zhang Y, Parikh A, Qian S. Migraine and stroke. Stroke Vasc Neurol 2017;2:160-7.

10. Sarchielli P, Granella F, Prudenzano MP, et al. Italian guidelines for primary headaches: 2012 revised version. J Headache Pain 2012;13 Suppl 2:31-70.

11. Evers S, Afra J, Frese A, et al. EFNS guideline on the drug treatment of migraine-revised report of an EFNS task force. Eur J Neurol 2009;16:968-81. 
12. Zhao L, Chen J, Li Y, et al. The long-term effect of acupuncture for migraine prophylaxis: a randomized clinical trial. JAMA Intern Med 2017;177:508-15.

13. Burke A, Upchurch DM, Dye C, et al. Acupuncture use in the united states: findings from the national health interview survey. J Altern Complement Med 2006;12:639-48.

14. Linde K, Allais G, Brinkhaus B, et al. Acupuncture for the prevention of episodic migraine. Cochrane Database Syst Rev 2016(6):CD001218.

15. Silberstein SD. Practice parameter: evidence-based guidelines for migraine headache (an evidence-based review): report of the quality standards subcommittee of the american academy of neurology. Neurology 2000;55:754-62.

16. Zhao ZQ. Neural mechanism underlying acupuncture analgesia. Prog Neurobiol 2008;85:355-75.

17. Han JS. Acupuncture and endorphins. Neurosci Lett 2004;361:258-61.

18. Huang C, Wang Y, Han JS, et al. Characteristics of electroacupuncture-induced analgesia in mice: variation with strain, frequency, intensity and opioid involvement. Brain Res 2002:945:20-5.

19. Goldman N, Chen M, Fujita T, et al. Adenosine A1 receptors mediate local anti-nociceptive effects of acupuncture. Nat Neurosci 2010;13:883-8.

20. Zhao LP, Liu L, Pei P, et al. Electroacupuncture at Fengchi (GB20) inhibits calcitonin gene-related peptide expression in the trigeminovascular system of a rat model of migraine. Neural Regen Res 2017;12:804-11.

21. Li Z, Liu M, Lan L, et al. Altered periaqueductal gray resting state functional connectivity in migraine and the modulation effect of treatment. Sci Rep 2016;6:20298.

22. Vickers AJ, Cronin AM, Maschino AC, et al. Acupuncture for chronic pain: individual patient data meta-analysis. Arch Intern Med 2012:172:1444-53.

23. Linde K. Evidence of effectiveness but not efficacy - why many complementary therapies are so hard to accept for biomedicine. BMC Complementary and Alternative Medicine 2017;17:S3.

24. Vickers AJ, Vertosick EA, Lewith G, et al. Acupuncture for chronic pain: update of an individual patient data meta-analysis. J Pain 2018;19:455-74.
25. Ad Hoc Committee on the Classification of Headache. Classification of headache. Arch Neurol 1962;6:173-6.

26. Headache Classification Subcommittee of the International Headache Society. The international classification of headache disorders: 2nd edition. Cephalalgia 2004;24:9-160.

27. Classification and diagnostic criteria for headache disorders, cranial neuralgias and facial pain. Headache Classification Committee of the International Headache Society. Cephalalgia 1988;8:1-96.

28. MacPherson H, Altman DG, Hammerschlag R, et al. Revised standards for reporting interventions in clinical trials of acupuncture (stricta): extending the consort statement. PLoS Med 2010;7:e1000261.

29. Wu XK, Stener-Victorin E, Kuang HY, et al. Effect of acupuncture and clomiphene in chinese women with polycystic ovary syndrome: a randomized clinical trial. JAMA 2017;317:2502-14.

30. Liu Z, Liu Y, Xu H, et al. Effect of electroacupuncture on urinary leakage among women with stress urinary incontinence: a randomized clinical trial. JAMA 2017;317:2493-501.

31. Li Y, Zheng H, Witt CM, et al. Acupuncture for migraine prophylaxis: a randomized controlled trial. CMAJ 2012;184:401-10.

32. Streitberger K, Kleinhenz J. Introducing a placebo needle into acupuncture research. Lancet 1998;352:364-5.

33. Tfelt-Hansen P, Pascual J, Ramadan N, et al. Guidelines for controlled trials of drugs in migraine: third edition. A guide for investigators. Cephalalgia 2012;32:6-38.

34. In: Higgins JPT, Green S, eds. Cochrane Handbook for Systematic Reviews of Interventions Version 5.2.0. 2017: The Cochrane Collaboration. www.cochrane-handbook.org.

35. Brown SA, Upchurch SL, Acton GJ, et al. A framework for developing a coding scheme for meta-analysis. West $J$ Nurs Res 2003;25:205-22.

36. Wolfgang V. Conducting Meta-Analyses in $\mathrm{R}$ with the metafor Package. Journal of Statistical Software 2010;36:1-48.

37. Schwarzer G, Carpenter JR, Rücker G. Meta-Analysis with R (Use-R!). Switzerland: Springer International Publishing, 2015. http:// www.springer.com/gp/book/9783319214153

38. Schünemann $\mathrm{H}$, Brożek J, Guyatt $\mathrm{G}$, et al. GRADE handbook for grading quality of evidence and strength of recommendations. http:// www.guidelinedevelopment.org/handbook (accessed 20 Mar2014). 